\title{
Las historias críticas de la Psicología desde la perspectiva de género. Pensando sus influencias y posibilidades
}

\section{Histórias críticas da Psicologia a partir da perspectiva de gênero. Pensando sobre suas influências e possibilidades.}

The critical history's of Psychology from a gender perspective. Thinking influences and possibilities.

\author{
Ana Elisa Ostrovsky* \\ Consejo Nacional de Investigaciones Científicas y Técnicas - \\ CONICET, Universidad Nacional de Mar del Plata, Mar del Plata, \\ Argentina
}

\begin{abstract}
RESUMEN
En el presente trabajo queremos mostrar elementos que propiciaron el desarrollo de la historia de la psicología desde la perspectiva de género en las últimas décadas. Nuestra hipótesis es que el origen de dicha perspectiva se relaciona con la renovación de la historia tradicional de la psicología, el consecuente florecimiento de la historia crítica de la psicología, el surgimiento de la categoría género en ciencias sociales, y el nacimiento de la historia de las mujeres y la historia de género en las humanidades. Para organizar la exposición de nuestro trabajo, desarrollaremos cada uno de dichos elementos para luego ofrecer un estado del arte de los trabajos en historia de la psicología desde una mirada de género.
\end{abstract}

Palabras clave: Historia de la psicología, Género, Perspectivas críticas.

\section{RESUMO}

Neste artigo apontaremos os elementos que levaram ao desenvolvimento da história da psicologia a partir da perspectiva do género nas últimas décadas. Nossa hipótese é que a origem dessa perspectiva está relacionada com a renovação da história tradicional da psicologia, o subsequente florescimento da história crítica da psicologia, o aparecimento da categoria de gênero em ciências sociais, e o nascimento da história mulheres e história de gênero na área de ciências humanas. Organizamos a exposição do nosso trabalho desenvolvendo cada um desses itens e em seguida, oferecendo o estado da arte em história da psicologia a partir de uma perspectiva de gênero.

Palavras-Chave: História da psicologia, Gênero, Perspectivas críticas.

\section{ABSTRACT}

In the present work we want to show elements that led to the development of the history of psychology from gender perspective in recent decades. Our hypothesis is that the birth of that perspective is related to the renewal of 
the traditional history of psychology, the subsequent flowering of the critical history of psychology, the emergence of the gender category in social science, and the birth of history women and gender history in the humanities. To organize the exhibition of our work, develop each of these items and then offer a state of the art work in history of psychology from a gender perspective.

Keywords: History of psychology, Gender, Critical perspectives.

\section{Introducción}

En las últimas décadas hemos visto florecer diversos trabajos que emplean la perspectiva de género en historia de la psicología. Aunque difieren en sus unidades de análisis, su tradición historiográfica, e inclusive en la ponderación de su impacto en la historiografía tradicional, tales estudios testimonian la creciente preocupación por examinar críticamente las teorías y prácticas psicológicas a la luz de la categoría género y sus posibilidades para reconstruir el pasado disciplinar y enfocar el futuro desde otra mirada.

Para analizar su campo y posibilidades de aplicación, mostraremos elementos que a nuestro entender propiciaron el desarrollo de la historia de la psicología desde la perspectiva de género en las últimas tres décadas. Nuestra hipótesis es que el origen de la perspectiva se encuentra fuertemente relacionado con tres fenómenos. En primer lugar, con la renovación de la historia tradicional de la psicología de la mano de los enfoques críticos, luego con el surgimiento de la categoría género en ciencias sociales, y en tercer lugar, con el surgimiento de la historia de las mujeres y la historia de género en las humanidades.

En dicho marco, el objetivo de nuestro trabajo es desarrollar brevemente cada uno de los tres ejes propuestos, para luego ofrecer un panorama general de los trabajos en historia de la psicología que emplearon la categoría género subrayando sus potencialidades.

\section{La historia crítica de la psicología}

Ubicamos el surgimiento de la historia crítica de la psicología en el cambio de enfoque acaecido dentro de la historiografía de la psicología durante el lapso temporal de las décadas de los setenta y ochenta del siglo XX. En estas décadas se produjo la consolidación de la profesionalización del historiador de la disciplina de la mano de un viraje en su manera de abordar el pasado, fenómeno que se tradujo en su énfasis en autores y autoras otrora descuidados, su ruptura con la dicotomía interno- externo, su posición crítica ante la inmutabilidad de los objetos psicológicos, y su creciente relación con la historiografía general y la historiografía de las ciencias entre otras cuestiones. (DANZIGER, 1984; MASSIMI, 1996; ROSE, 1996) 
Dicho viraje, que modificó el desalentador panorama para la disciplina presentado por Robert Watson (1960), se enmarcó en los cambios generales producidos en la década del setenta. Tal periodo se caracterizó en el campo de las ciencias sociales y humanas por un profundo cambio epistemológico en la manera de concebir la ontología de los objetos de estudio. Se profundizó la crítica al naturalismo ingenuo, se consolidó la caída de la ilusión del progreso científico lineal, y cada vez más surge la conciencia de la reflexividad $^{1}$ como una peculiaridad del abordaje de lo humano (VERA FERNÁNDEZ, 2008).

Todas ésta fracturas abonaron los debates de las disciplinas psicológicas que cuestionaron su propio modo de historiar su pasado. En dicho marco comenzó a pensarse los métodos y los objetivos de la historia de la psicología y su particular relación con la psicología misma. La historia de la psicología tradicionalmente había sido practicada por psicólogos aficionados al campo y con un espíritu legitimante respecto a los conocimientos actuales. Se trataba de una historia destinada principalmente a estudiantes de psicología cuya función era proyectar, a través de sus hitos y su galería de autores, una suerte de identidad profesional oficial para el novel aspirante. Este ejercicio socializador, cuya función era establecer tradiciones historiográficas portadoras de coherencia, filiación y legitimidad, generalmente cometía las falacias históricas del presentismo y el justificacionismo como se observa paradigmáticamente en los trabajos pioneros de Edwin Garrigues Boring (BORING; LANGFELD; WELD, 1935, 1937, 1939, 1948). Ello se promovía al establecer líneas espurias de ascendencia, interpretaciones del pasado en virtud de las certezas del presente, y estrategias que servían para respaldar los lugares conquistados en el juego de poderes propio de todo campo de saber. (ASH, 1983; GOODWIN, 1997; KLAPPENBACH, 2006).

Respecto al presentismo, quedaba claro a los nuevos historiadores de la psicología que toda investigación sobre el pasado inevitablemente partía del presente y los problemas actuales que la motivaban. El punto era entonces más que intentar emprender la imposible tarea de distanciarse del presente, el procurar no definir al mismo como menos conflictivo o más "maduro" que el pasado disciplinar. (VILANOVA, 1997). Otra reflexión se desprendía también del particular posicionamiento de la historia de la psicología como un objeto complejo que por su pluralidad de tradiciones científicas, objetos, métodos, técnicas y usos, demandaba una historia conectada con ciencias sociales y humanas, pero también con las ciencias naturales y las disciplinas formales. En efecto, se produjo la creciente conciencia de que la historia de la psicología podía dirigir sus 
preguntas hacia aspectos tan distintos como la genealogía de sus conceptos, sus diversos usos tecnológicos, sus procedimientos de profesionalización y las modalidades concretas de su implatación cultural (VEZZETTI, 2007). Asimismo se advirtió que dicho carácter de ciencia puente (VILANOVA, 1996) simultáneamente facilitaba y dificultaba la tarea del especialista. Por un lado le permitía disponer de un variado repertorio de herramientas analíticas oriundas de saberes afines que lo enriquecían y lo alejaban del dogmatismo, pero simultáneamente lo comprometía con la exigencia de profundidad y especialidad ajustadas al propio campo (KLAPPENBACH, 2000).

Es entonces que en estas décadas, de la mano de la mencionada profesionalización, se observó un acercamiento entre la historia de la psicología y la historia de las ciencias en general, perfeccionándose de ésta manera las metodologías de estudio que se volvieron más rigurosas respecto a los cánones de la historia general. La historia de la psicología así concebida se volvió más externa y los nuevos historiadores lograron la producción de un conocimiento valioso alejado del modelo de historia whig autocomplaciente de la historia interna, pero pagando con ello el precio de estar aislados de la comunidad científica de los psicólogos. El desafío para Kurt Danziger (1993), uno de sus más eximios representantes, es que ésta historia del historiador profesional tenga impacto en la psicología misma, ya que si que los objetos de la ciencia son objetos históricos, entonces la historia podría reclamar legítimamente un lugar jerarquizado a la hora de estudiar científicamente los mismos. La historia de la psicología desde tal posición, participaría del debate abierto con el psicólogo especializado en su propio dominio de investigación, actuando como una teoría que enuncia la historicidad de la propia psique, aportando explicaciones históricas sobre los productos de la investigación científica, su otorgamiento de valor y la organización social de la comunidad científica. (ROSA RIVERO, 2008).

Siguiendo lo enunciado, ubicamos en la década de los noventa la pregunta por la relación entre la historia interna y la historia externa, y en dicho marco la procedencia disciplinar y la formación del historiador de la psicología. Se trataba de reconocer el carácter histórico del historiador de la psicología en sus herramientas de investigación, pero sin dejar de lado la cuestión del destino ${ }^{2}$ de sus investigaciones y su impacto directo en la comunidad científica de los psicólogos, escapando de la disyuntiva entre una historia externa crítica o una historia interna celebratoria (KLAPPENBACH, 2002).

La historia de la psicología se relaciona entonces con los psicólogos no para complacerlos ni otorgarles una identidad, como temía Joseph Brozek (1980) respecto a los enfoques internistas, sino para aportar elementos críticos de debate para repensar su pasado, entender su 
presente y proyectar su futuro, poniendo en dicho movimiento la historia al servicio de la psicología, pero sin subordinarla a ella ni a sus objetivos. (BROCK, 1995; ROSA; HUERTAS; BLANCO, 1998)

\section{El surgimiento de la categoría género}

Todas estas transformaciones acaecidas desde los años setenta también coinciden con la segunda ola del feminismo, los estudios de historia de las mujeres y la consolidación de la categoría género en ciencias sociales. La Segunda Ola de Feminismo, que tuvo como antecedente al Segundo sexo de Simone de Beauvoir de 1949, floreció a finales de los años 60 y principios de los 70, principalmente en Estados Unidos y en Europa, con el lema "Io personal es político". Los ejes principales de su crítica radicaban en la toma de conciencia de que todas las esferas de la vida social estaban estructuradas por relaciones de poder en las cuales las mujeres aparecían como subordinadas a los hombres. (CANGIANO; DU BOIS, 1993). La categoría género es solidaria a dicho movimiento y se utilizó para deconstruir al término sexo en el terreno académico de las ciencias sociales. Efectivamente aportes de la sociología, la antropología, la historia, entre otras, enfocaron sus novedosos estudios de género entendiendo al mismo como una categoría socialmente construida que se imponía a un cuerpo biológico y la emplearon en los espacios académicos abiertos por los estudios de mujeres (NAVARRO; STIMPSON, 1998).

Específicamente al referirse al género la literatura sobre el tema toma como hito sistematizador al artículo de la historiadora Joan Scott Gender a Useful Category Of Historical Analysis de 1988. En dicha publicación, la autora desplaza el concepto universalista, ahistórico y esencialista de "mujer" por el de género, para subrayar que el saber sobre la diferencia sexual se conforma históricamente y ordena las relaciones sociales en "una categoría social que se impone en un cuerpo sexuado “( $p$. 22). A su vez, se acentúa que la categoría presenta un carácter relacional en tanto lo masculino y lo femenino se conforman a partir de una relación mutua en un momento histórico dado, siendo una forma primaria de relaciones significantes de poder. Al analizar la definición de Scott, Ana María Bach, María Luisa Femeninas, Alicia Gianella, Margarita Roulet e Isabel Santa Cruz (1994) extraen cuatro elementos relacionados: símbolos culturales que evocan representaciones, conceptos normativos, instituciones que reproducen y refuerzan dichos conceptos, y la identidad subjetiva. Dichos elementos se manifestarían como indisociables de las relaciones de poder. Por tal razón, al intentar conocer cualquier "objeto" se debería tener en cuenta tales determinaciones subrayadas por las epistemologías feministas, las 
cuales podrían pensarse como un heterogéneo conjunto de posturas que presentan como común denominador el cuestionamiento de la ciencia tradicional en tanto androcéntrica y la crítica de las bases epistemológicas y metodológicas que la sustentan. (HARDING, 1987, 1996; STOLKE, 2004; FERRARI, 2005; GUZMÁN; PÉREZ, 2007; MATOS, 2008).

En síntesis, podríamos entender al género como a un conjunto social de prácticas interrelacionadas que en un particular momento histórico definen a lo masculino, lo femenino y lo trans, estructurando relaciones de poder que se manifiestan tanto en las representaciones sociales y en las imágenes normativas, como en la identidad personal y las configuraciones vinculares de los sujetos. Tales prácticas guardarían una compleja relación con la biología, en la medida en que se construirían sobre el dato biológico de la diferencia sexual, pero también construirían como originario y prediscursivo a éste, despojándolo de su encarnadura social y naturalizando las diferencias como si éstas emanaran de la naturaleza.

Al postular lo antedicho, la perspectiva de género cambió la manera de enfocar la historia: la historia pretendidamente universal tuvo que incorporar la historia de las mujeres, y simultáneamente se vio compelida a definir el mismo término "mujer" en su construcción y colocación temporal.

\title{
Historia de las mujeres e historia del género: La renovación de la historia de las mujeres
}

\begin{abstract}
Cuando miramos sólo con un ojo nuestro campo de visión es limitado y carece de profundidad. Si miramos luego con el otro, nuestro campo visual se amplía pero todavía le falta profundidad. Sólo cuando abrimos los dos ojos a la vez logramos tener todo el campo de visión y una percepción exacta de la profundidad (LERNER, 1990 p. 20)
\end{abstract}

La historia de las mujeres surgió principalmente en Gran Bretaña, Estados Unidos y Francia en los años setenta. La idea fuerza de dicho movimiento fue por un lado el "restituir las mujeres a la historia" y por otro el "restituir la historia a las mujeres". Ciertamente se trataba de cuestionar una historia pretendidamente universal, pero motorizada por "grandes hombres" y escrita mayormente por varones, para dar paso a historias situadas que rescaten el aporte de las mujeres y su experiencia histórica. A su vez, la conciencia de alteridad y de desigualdad entre la historia femenina y la masculina tempranamente se complementó con la idea de diferencia y alteridad al conjunto de las mismas mujeres, razón por la cual desde sus inicios se esgrimió como una "historia de las mujeres" en plural 
subrayando dicha heterogeneidad. (PÉROTIN-DUMON, 2001).

Siguiendo a Michelle Perrot (2002), una de sus pioneras, la emergencia del objeto "mujeres" en las humanidades en general y en la historia en particular, fue propiciada por una serie compleja de factores. Por una parte encontramos los factores sociológicos y políticos como la presencia de las mujeres en la universidad, la creciente participación de mujeres en los espacios públicos, y la consolidación de movimientos feministas que iniciaron un trabajo de memoria y de recuperación de los aportes femeninos en distintas esferas sociales. Por otra parte, la emergencia del objeto mujeres fue favorecida por factores científicos relacionados con la crisis de los sistemas generales de pensamiento. Efectivamente el cuestionamiento de la capacidad omniexplicativa del marxismo y el estructuralismo como grandes paradigmas en historia, según la citada autora, produjeron una modificación de las alianzas disciplinarias. La historia en los años setenta se reconcilió poco a poco con la antropología, la sociología y empezó a redescubrir nuevos objetos de análisis. Por ésos años Perrot menciona un clima de ebullición de nuevos enfoques encarnados en la Historia de la Locura y la Historia de la Sexualidad de Michel Foucault y el desarrollo de "La Nueva Historia" en manos de la tercer generación de los Annales ${ }^{3}$. Dicha generación multiplicaba los objetos de indagación cambiando la tradicional manera de hacer y escribir historia. Como señaló Jacques Revel $^{4}$, uno de sus integrantes, ya no se trataba de mirar al pasado como una explicación del presente y una proyección del futuro siguiendo la lógica del gran relato, sino de mirar al pasado como el antropólogo mira a una cultura desconocida, desde los propios esquemas, pero como algo ajeno, con intrincados códigos y señas a la espera de ser comprendidas y descifradas por el historiador.

En ése marco de surgimiento de estudios sobre historia de la infancia, la familia, la vida privada, la belleza, la religiosidad, la microhistoria, etc., la historia de mujeres surgió aportando nuevas miradas y renovadas preguntas que se dirigían a incluir a las mujeres en el conjunto de las relaciones sociales estudiadas. Siguiendo a Georges Duby (1992), se trataba de pensarlas y representarlas en la medida en que ellas frecuentemente no se representaban a sí mismas: eran representadas desde la mirada masculina que se fijaba sobre ellas para reducirlas o seducirlas.

Dicha cuestión se observó en la propia escasez de fuentes, ya que sólo se sistematiza como legado aquello que resulta relevante en un determinado período histórico. En tal sentido, la historia de las mujeres ha tenido que afrontar el problema de las fuentes teniendo que valorar indicios, catalogar materiales, interpretar documentos y generar sus propios registros, ya que o faltaban textos de mujeres, o 
bien abundaban los textos sobre las mujeres plasmados de esencialismos, incomprensiones y caricaturizaciones sobre la feminidad (RAMOS, 2003)

Para compensar dicha debilidad el enfoque utilizó un pluralismo metodológico que le permitió incluir la biografía, la historia oral, aportes antropológicos, y herramientas propias de la historia económica, la historia política, la historia de las ideas, la historia de las mentalidades y la historia social. (BOCK, 1989). Los frutos de tal renovación se evidenciaron en obras claves como los cinco volúmenes de la obra Historia de las Mujeres en Occidente dirigida por George Duby y Michelle Perrot (1991), el trabajo de Bonnie Anderson y Judith Zinsser (1991) Historia de las mujeres: una historia propia, la compilación de Juan Andreo y Sara Guardia (2002) Historia de las mujeres en América Latina, la compilación de Fernanda Gil Lozano, Valeria Pita y María Gabriela Ini (2000) Historia de las Mujeres en la Argentina, y revistas especializadas como Journal of Women's History, Clío, Gender and History y Arenal entre otras.

También dicha historia empezó a deconstruir el plural "mujeres" y pensar al género en un salto que incorporó una mirada relacional e integradora capaz de analizar las relaciones entre los géneros en su totalidad y su correspondencia con lo biológico y lo social tal como lo planteó la antropóloga Gayle Rubin (1986) al acuñar la denominación sistema sexo-género. Michelle Perrot mencionó que con dicha incorporación la historia de las mujeres cambió en sus objetos de estudio y perspectivas de análisis. Fue ampliando su interés inicial por el cuerpo femenino y los roles privados propios de lo doméstico para estudiar a las mujeres en el espacio público de la ciudad, del trabajo, de la política, de la guerra y de la creación. A su parecer empezó por una historia de las mujeres para convertirse en una historia del género, que insiste sobre las relaciones entre los sexos e integra la masculinidad expandiendo sus perspectivas espaciales, religiosas y culturales. (PERROT, 2002).

La historia de las mujeres desde la mirada de género no es la historia de "la mitad de la humanidad" como afirmaban algunas feministas. Como consecuencia, los varones como grupo comenzaron a hacerse visibles en su faz de género, y surgieron interrogantes sobre la conformación de las relaciones entre los géneros desde diferentes perspectivas: la jerarquía, la dominación, la opresión, la división del trabajo, etc., cada una específica de cada contexto en la medida en que las conformaciones de género son históricas, variables y geográficamente situadas.

Otro de los conceptos empleados para explicar relaciones intergenéricas fue el del poder. Aunque hubo análisis sobre la dominación (BOURDIEU, 2000), o la explotación de las mujeres en 
manos de los hombres (DELPHI, 2001), el concepto de poder, particularmente en su conceptualización desde la microfísica del poder foucaultiana, fue empleado por los estudios históricos desde la perspectiva de género por su capacidad para explicar su ejercicio y circulación en situaciones cotidianas. Al decir del propio Michel Foucault:

\begin{abstract}
Entre cada cuerpo social, entre un hombre y una mujer, entre un maestro y su alumno, entre el que sabe y el que no sabe, pasan relaciones de poder que no son la proyección pura y simple del gran Poder soberano sobre los individuos; son más bien el suelo movedizo y concreto sobre el que ese poder se incardina, las condiciones de posibilidad de su funcionamiento (FOUCAULT, 1992, p. 160)
\end{abstract}

El uso del género dentro de ésta concepción permitió, siguiendo a Scott (1988), repensar los determinantes de las relaciones entre los sexos, no sólo como lo que les ocurrió a los hombres y a las mujeres en el pasado y la manera en la que ellos se relacionaron, sino la dinámica de ésa relación en el marco de la significación subjetiva y colectiva que una sociedad le da a lo masculino y a lo femenino, y como al hacerlo, se conforman identidades, cuerpos y ejercicios diversos de circulación del poder. El carácter sociocultural del género, su conformación histórica, su imaginería y su inserción cultural, también enriquecieron el cuestionamiento de la misma biología y las interpretaciones naturalistas del cuerpo. No se trataba de pensar que el sexo era un dato natural, sino contemplar como las concepciones de género definen una historia del cuerpo, una historia de la biología y una historia de las herramientas con las que los seres humanos representan y en ocasiones crean sus cuerpos y sus goces.

Asimismo, el enfocar la historia desde la mirada de género, implicaba correrse del principal riesgo de la historia de las mujeres: el quedar como un capítulo subordinado dentro de la historiografía, una mirada complementaria y acotada a lo femenino como grupo de interés. Otra ventaja del género era poder salir del modelo binario del dimorfismo sexual y estudiar históricamente las subversiones a ése orden y los procedimientos históricos de normalización, explicación de lo intersexual y lo transexual en sus múltiples manifestaciones. (HALPERÍN; ACHA, 2000).

El género entonces ha sido y sigue siendo un concepto nutricio en historia, y el diálogo entre la historia de las mujeres y la historia del género se produce en ocasiones en el marco de fronteras difíciles de establecer. Hay muchas epistemologías feministas y muchas maneras de entender al género, lo cual permite cierta porosidad entre lo que podemos establecer como historia de género, historia feminista e historia de las mujeres. Con todo, consideramos que la inclusión de 
la categoría género ha representado una importante contribución, pero más allá de eso coincidimos con Barrancos (2005) en que lo esencial no es la denominación del tipo de historia que se hace sino la clave de interrogación: las tramas categoriales que se emplean para pensar y reconstruir la acción humana sexualizada.

\section{La historia de la psicología desde la perspectiva de género}

Consideramos que las tres coordenadas mencionadas: el enfoque crítico, la categoría género, y la historia del género, han abonado notablemente el suelo desde el cual emergieron en los años setenta los primeros desarrollos de la historia de la psicología genéricamente orientada.

En Estados Unidos, debemos destacar la relevancia de los trabajos pioneros de Florence Denmark (1979, 1980, 1988, 1992, 1994) quien ha analizado la presencia y la representatividad femenina en las universidades, los sesgos sexistas en la investigación psicológica, y la presencia de estereotipos de género en los materiales didácticos destinados a estudiantes de psicología. La autora contribuyó además a la visibilización del campo desde su rol de quinta mujer presidenta de la APA entre 1980 y 1981 (DENMARK, 1988b) y desde allí organizó homenajes a psicólogas de renombre, publicaciones conjuntas sobre la participación femenina, y diversas estrategias de articulación entre historia, formación y socialización de los psicólogos sensibles a la perspectiva de género.

Similar lineamiento se vislumbró en posteriores aportes que problematizaron diversas temáticas de género que van desde la presentación de mujeres no contempladas en la "historia oficial" de la psicología, hasta el análisis de teorías y prácticas psicológicas fomentadoras de determinados roles de género a lo largo del desarrollo disciplinar. (FURUMOTO; SCARBOROUGH, 1986; LESLEY, 1986; PUENTE; MATTHEWS; BREWER, 1992; HOTHERSALL, 1997; WENTWORTH, 1999; KRESSLEY-MBA; JAEGER, 2003; KENDLER, 2003; HOOGLAND NOON, 2004; CAMERON; HAGEN, 2005; SHIELDS; STEPHANIE, 2007; JOHNSTON; JOHNSON, 2008; GOLDEN, 2008; PETTIT, 2008)

En Alemania es dable mencionar los estudios bibliométricos de Sprung (1996) los cuales mostraron la evolución de la participación femenina en los congresos de psicología según temáticas, a la vez que desarrollaron un modelo de aproximación de la mujer al trabajo científico según su relación de amistad, discipulado o parentesco con algún investigador de renombre. Dicha modelización resultó interesante para analizar los movimientos complejos y muchas veces paradojales de las mujeres en su inserción académica y profesional.

Dentro del mundo hispanohablante han empleado la perspectiva 
diversos grupos de trabajo. Cabe destacar en España a los aportes del Instituto de Estudios de la Mujer de Valencia (BARBERA HEREDIA, 2008; PASTOR; CIVERA; TORTOSA, 2000) sobre la evolución de los estudios de género y las relaciones sexo-género en la historia de la psicología, y los estudios de diversas autoras que rescataron críticamente el aporte de mujeres a la disciplina tanto en España como en otros puntos geográficos señalando mecanismos de exclusión y estrategias de resistencia (HERRERO, 1997, 2003; GARCIA DAUDER, 2005; GIMÉNEZ, 2007; GARCÍA COLMENARES, 2008; GIMÉNEZ SEGURA, 2008; PAZZIANI, 2008; PEÑARANDA ORTEGA; QUIÑONES VIDAL, 2008). Hacia un destino más amplio que el de la comunidad de los psicólogos, Esperanza Fiol y Victoria Ferrer Pérez, de la Universidad de Cadiz, rastrearon conceptos psicológicos en el interjuego de poderes políticos propios del espacio social de su emergencia (BOSCH FIOL; FERRER PÉREZ, 1998, 2008). De ésta manera se analizaron tópicos como la transmisión de estereotipos de género durante el franquismo y el perfil psicológico de las mujeres republicanas de acuerdo a los científicos de la época, temáticas que evidencian las relaciones entre los roles políticamente deseables para los géneros y la función legitimante y constructiva de la psicología en relación a los mismos.

Particularmente en Latinoamérica, además del surgimiento de distintos espacios sobre la materia ${ }^{5}$, han utilizado principalmente la perspectiva de género las investigaciones de María Inés Winkler de la Universidad de Santiago, en Chile (USACH) y Ana Jacó-Vilela de la Universidad del Estado de Rio de Janeiro (UERJ) (WINKLER 2001, 2004, 2007; JACÓ-VILELA, 2007, 2008). Las autoras analizaron la presencia de mujeres en los primeros laboratorios y espacios de psicología aplicada, como así también en los inicios de la psicología como profesión. Conjuntamente con la incorporación de figuras omitidas o secundarizadas en la historia de la psicología, avanzaron en el análisis de las paradojas en la autoría de los trabajos académicos y en el examen de los perfiles psicológicos y socioeducativos de tales mujeres. Otros aportes relevantes se observan en el estudio biográfico de Helena Antipoff (FREITAS CAMPOS, 2002), en el análisis del discurso psicológico sobre la maternidad y las diferencias de género (OLIVEIRA, 2006; CATHARINO, 2006), y en la aplicación de categorías provenientes del feminismo a la periodización del desarrollo histórico de la psicología (SAAVEDRA; NOGUEIRA, 2006).

Este listado, que no pretende ser exhaustivo y espera en el futuro enriquecerse con más aportes, testimonia no obstante la diversidad de posibilidades que abre el empleo de la mirada de género en la historia de de la psicología. Consideramos que tanto en la historia en 
general, como en la propia historia disciplinar, las perspectivas "criticas" cuestionaron las miradas cristalizadas y abrieron paso a la interrogación del género, interrogantes que al plantearse desde distintos marcos, mostraron el potencial heurístico del enfoque.

Reflexionamos también que la mirada de género es particularmente interesante al historiar un campo altamente feminizado como la psicología; una disciplina con aulas y asociaciones profesionales con fuerte presencia de mujeres, y con un entretramado práctico y discursivo que no siempre contempló dicho fenómeno y en ocasiones estuvo encarnado en "maestros", "autores" y "mentores" reproductores de los roles tradicionales de género.

Con todo, en su conjunto, las primeras preguntas que la comunidad se fue planteando abrieron un movimiento histórico reparador centrado en rescatar y ponderar variadas trayectorias femeninas olvidadas o desestimadas. Creemos que con la consolidación de la perspectiva, dicho movimiento que fue y continúa siendo necesario, se complementará con estudios que, desbordando lo propiamente femenino, analicen el histórico papel de psicología en la construcción de la masculinidad hegemónica y en la esencialización y patologización de los diversos géneros. Pensamos que estas cuestiones serán cardinales en una historia capaz de pensar sus propios sesgos al mirar su pasado, intervenir en la psicología del presente, y proyectar su futuro como campo especializado.

\section{Referencias}

ANDERSON, B.; ZINSSER, J. Historia de las mujeres: una historia propia. Barcelona: Crítica, 1991.

ANDREO, J.; GUARDIA, S. Historia de las mujeres en América Latina. Murcia: Centro de Estudios La Mujer en la Historia de América Latina, CEMHAL, 2002.

ASH, M. The self-presentation of a discipline: History of psychology in the United States between pedagogy and scholarship. En GRAHAM, L.; LEPENIES, W.; WEINGART, P. (Eds.). Functions and uses of disciplinary histories. Boston: D. Reidel, 1983, p. 143-189.

BACH, A; FEMENÍAS, L.; GIANELLA, A.; ROULET, M.; SANTA CRUZ, I. Para comprender el género: Precisiones epistemológicas. En SANTA CRUZ, I.; BACH, A.; FEMENÍAS, L.; GIANELLA, A.; ROULET, M. Mujeres y Filosofía (I). Teoría filosófica de género. Buenos Aires: Centro Editor de América Latina, 1994, p. 59-66.

BARBERÁ HEREDIA, E.; CALA CARRILLO, J. Desarrollo histórico en la investigación psicológica del enfoque de género. Revista de Historia de la Psicología, v. 29 n. 3/4, p. 25-33, 2008.

BARRANCOS, D. Historia, historiografía y género. Notas para la memoria de sus vínculos en la Argentina. Aljaba, Luján, p. 9:49-72, 
2005.

BOCK, G. La historia de las mujeres y la historia del género: Aspectos de un debate internacional. Historia Social, v. 9, p. 55-77, 1989.

BORING, E.; LANGFELD, H.; WELD, H. (Eds.). Psychology: A factual textbook. New York/London: J. Wiley \& Sons/Chapman \& Hall, 1935.

BORING, E.; LANGFELD, H.; WELD, H. (Eds.). A manual of Psychological experiments. New York/London: J. Wiley \& Sons/ Chapman \& Hall, 1937.

BORING, E.; LANGFELD, H.; WELD, H. (Eds.). Introduction to Psychology. New York/London: J. Wiley \& Sons/Chapman \& Hall, 1939.

BORING, E.; LANGFELD, H.; WELD, H. (Eds.). Foundations of Psychology. New York: J. Wiley, 1948.

BOSCH FIOL, E.; FERRER PEREZ, V. La transmisión del estereotipo femenino a traves de la historia: El caso de España y el papel de la Seccion femenina de Falange Española y de las Jons. Revista de Historia de la Psicología, v. 19, n. 2/3, p. 339-404, 1998.

BOSCH FIOL, E.; FERRER PÉREZ, V.; NAVARRO GUZMÁN, C. La psicología de las mujeres republicanas según el Dr. Antonio Vallejo Nájera. Revista de Historia de la Psicología, v. 29, n. 3/4, p. 3540, 2008.

BOURDIEU, P. La dominación masculina. Barcelona: Anagrama, 2000.

BROCK, A. Why I am not a Historian of Science. History and Philosophy Bulletin, v. 7, n. 2, p. 27-31, 1995.

BROZEK, J. ET.AL. Historiografy of Modern Psychology. Toronto: Hofrege, 1980.

CAMERON, C.; HAGEN, J. Women in child development: Themes from the SRCD Oral History Project. History of Psychology, v. 8, n. 3, p. 289-316, 2005.

CANGIANO, M.; DU BOIS, L. De mujer a género: teoría interpretación y practica feminista en las ciencias sociales. Buenos Aires: Centro Editor de América Latina, 1993.

DANZI GER, K. Towards a Conceptual Framework for a Critical History of Psychology. Revista de Historia de la Psicología, v. 5, n. 1/2, p. 99-107, 1984.

DANZIGER, K. Tres desafíos para la historia de la psicología. Buenos Aires: Facultad de Psicología, Conferencia, División 26 de la APA, Toronto, agosto de 1993. Disponible en: <http://www.elseminario.com.ar/>. Acceso: 10 nov. 2009.

DELPHY, C. L’Ennemi principal: Pensar le genre. Paris: Nouvelles Questions Féministes, 2001.

DENMARK, F. Women in psychology in the United States. Annals of the New York Academy of Science, v. 323, p. 65-78, 1979. 
DENMARK, F. Psyche: from rocking the cradle to rocking the boat. American Psychologist, v. 35, p. 1057-1065, 1980.

DENMARK, F. and col. Guidelines for Avoiding Sexism in Psychological Research. A Report of the Ad Hoc Committee on Nonsexist Research.

American Psychologist, v. 43, p. 582-585, 1988.

DENMARK, F. Autobiography. En O'Connell, A.; Russo, N. (Eds.). Models of achievement: Reflections of eminent women in psychology Vol. 2, Hillsdale: Erlbaum, 1988b, p. 279-294

DENMARK, F.; FERNANDEZ, L. Women: Their influence and their impact on the Teaching of Psychology. En: PUENTE, A.; MATTHEWS, J.; BREWER, C. (comp) Teaching Psychology in America: a history. Washington: APA, 1992, p. 121-160.

DENMARK, F. Engerending Psychology. American Psychologist, v. 49, p. 329-334. 1994.

DUBY, G. I mages de femmes. París: Plon, 1992.

DUBY, G.; PERROT, M. (Dir.). Historia de las Mujeres en Occidente. Madrid: Taurus, 1991.

CAMPOS, R. H. F. (Org.). Helena Antipoff, Textos Escolhidos. São Paulo: Casa do Psicólogo, 2002.

FERRARI, L. Acerca de los silencios críticos de la ciencia. Contribuciones de las epistemologías feministas a las relaciones entre ciencia, sociedad y género. Revista Subjetividad y Procesos Cognitivos, v. 7, p. 90-104, 2005.

FOUCAULT, M. Microfísica del poder. Madrid: Ediciones la Piqueta, 1992.

FURUMOTO, L.; SCARBOROUGH, E. Placing women in the History of Psychology. American Psychologist, v. 41, p. 35-42, 1986.

GARCÍA COLMENARES, C. Las primeras psicólogas españolas becadas por la Junta de Ampliación de Estudios e Investigaciones Científicas. Revista de Historia de la Psicología, v. 29, n. 3/4, p. 69-76, 2008.

GARCIA DAUDER, S. Psicología y feminismo. Historia olvidada de mujeres pioneras en Psicología. Madrid: Narcea, 2005.

GIL LOZANO, F.; PITA, V.; INI, M. Historia de las mujeres en Argentina. Buenos Aires: Taurus, 2000.

GIMÉNEZ SEGURA, M. Elizabeth Fry (1780-1845) y Dorothea Dix (1802-1887): dos pioneras del tratamiento moral. Revista de Historia de la Psicología, v. 29, n. 3/4, p. 85-91, 2008.

GIMÉNEZ, M. Las mujeres en la Historia de la Psicología. Revista de Historia de la Psicología, v. 28, n. 2/3, p. 281-290, 2007.

GOLDEN, R. William Osler's "The nervousness of American women." History of Psychology, v.11, n. 1, p. 1-14, 2008.

GOODWIN, J. The vital role of psychology's history in introductory courses: An interview with Ludy T. Benjamin. Teaching of 
Psychology, v. 24, n. 3, p. 218-221, 1997.

GUZMÁN, M.; PÉREZ, A. Teoría de Género y Demarcación Científica. Cinta Moebio, v, 30, p. 283-295, 2007.

HALPERÍN, P.; ACHA, O. Historia de las mujeres e historia del género. En Acha, O.; Halperín, P. (2000). (comp.). Cuerpos, géneros e identidades. Estudios de Historia de género en Argentina. Buenos Aires: Ediciones del Signo, 2000, p. 11-28.

HARDING, S. Feminism and Methodology. Bloomington/ Indianapolis: Indiana University Press, 1987.

HARDING, S. El género y la ciencia: dos conceptos problemáticos. En su Ciencia y feminismo. Madrid: Morata, 1996, p. 28-51.

HERRERO, F. La escuela de Ginebra en la psicología aplicada española: la figura de Mercedes Rodrigo. Revista de Historia de la Psicología, v, 18, n. 1-2, p. 139-149,1997.

HERRERO, F. Mercedes Rodrigo (1891-1982), la primera psicóloga española. Revista de Psicología General y Aplicada , v. 56, n. 2, p. 139-148, 2003.

HOOGLAND NOON, D. Situating gender and professional identity in american child study, 1880-1910. History of Psychology, v. 7, n. 2, p. 107-129, 2004.

HOTHERSALL, D. Historia de la psicología. Mexico: Mac Graw Hill, 1997.

J ACÓ-VILELA, A.; SANTOS, V. F.; SILVA, L. L. Mulheres na Psicologia: os Arquivos Brasileiros de Psicotécnica. En Anais do XIV Encontro Nacional da Abrapso. Diálogos em Psicologia Social, Rio de Janeiro, UERJ, 2007, p. 1-12.

JACÓ-VILELA, A.; SANTOS, V. F.; BARBOSA, C. Mulheres do Laboratório de Biologia Infantil. Boletim do CDPHA, v. 20, p. 8992, 2008.

JOHNSTON, E.; JOHNSON, A. Searching for the second generation of American women psychologists. History of Psychology, v. 11, n. 1, p. 40-72, 2008.

KENDLER, T. A woman's struggle in academic psychology (1936 2001). History of Psychology, v. 6, n. 3, p. 251-266, 2003.

KLAPPENBACH, H. Historia de la historiografía de la psicología. En J. C. RíOS; R. RUIZ; J. C. STAGNARO; P. WEI SMMANN (Eds.). Psiquiatría, psicología y psicoanálisis. Historia y Memoria, Buenos Aires: Polemos, 2000, p. 238-259.

KLAPPENBACH, H. Historiadores internos y externos. La finalidad de la Historia de la Psicología. Actualidad Psicológica, v. 27, n. 294, p. 5-8, 2002.

KLAPPENBACH, H. Construcción de tradiciones historiográficas en psicología y psicoanálisis. Psicologia em Estudo, v. 11, n. 1, p. 317, 2006. 
KRESSLEY-MBA R.; JAEGER, S. Rediscovering a missing link: The Sensory Physiologist and Comparative Psychologist Mathilde Hertz (1891-1975). History of Psychology, v. 6, n. 4, p. 379-396, 2003. LERNER, G. La creación del patriarcado. Barcelona: Crítica, 1990. LESLEY, A. The Paradox of G. Stanley Hall Foe of Coeducation and Educator of Women. American Psychologist, v. 41, n. 8, p. 868878, 1986.

MASSIMI, M. Historiography of psychology: old and new paths. Cuadernos Argentinos Historias de la Psicología, v. 2, n. 1/2, p. 95-107, 1996.

MATOS, M. Teorias de gênero ou teorias e gênero? Se e como os estudos de gênero e feministas se transformaram em um campo novo para as ciências. Revista Estudos Femeninos, v. 16, n. 2, p. 333357, 2008.

OLIVEIRA, F. M. A revista nova, o discurso psicológico e a subjetividade feminina. En: JACÓ VILELA, A.; CARLOS CEREZO, A.; CONDE DE BARROS RODRIGUES, H. (Org.) Clio-PsychéSubjetividae e História. Juiz de Fora: Clio Edições Eletrônicas, 2006, p. 104-110.

NAVARRO, M.; STIMPSON, C. (comp). ¿Qué son los estudios de las mujeres? Buenos Aires: Fondo de Cultura Económica, 1998.

PASTOR, J.; CIVERA, C.; TORTOSA, F. La mujer en la Escuela Berlinesa de Psicología de la Gestalt: ¿Espacio cedido o conquistado?, Wera Mahler (1899-1991): Una psicóloga alemana y un ejemplo de tesón ante la adversidad. Revista de Historia de la Psicología, v. 21, n. 2-3, p. 597-612, 2000.

PAZZIANI, A. Charlotte Bühler's pioneering experimental study of infants' attitudes to novelty. Revista de Historia de la Psicología, v. 29, n. 3/4, p. 153-161, 2008.

PEÑARANDA ORTEGA, M.; QUIÑONES VIDAL, E. La figura de María Eugenia Romano Pérez en el desarrollo de la psicología española. Revista de Historia de la Psicología, v. 29, n. 3/4, p. 163-169, 2008.

PÉROTIN-DUMON, A. (Ed.). El género en historia. Londres: Institute of Latin American Studies, University of London, 2001.

PERROT, M. Mi historia de las mujeres. Buenos Aires: Fondo de Cultura Económica, 2008.

PETTIT, M. The new woman as "tied-up dog": Amy E. Tanner's situated knowledges. History of Psychology, v. 11, n. 3, p. 145$163,2008$.

PUENTE, A.; MATTHEWS, J.; BREWER, C. (Eds.). Teaching Psychology in America: a History. Washington: APA, 1992.

RAMOS, D. Historia de las mujeres, saber de las mujeres: la interpretación de las fuentes en el marco de la tradición feminista. 
Feminismo/ s, v. 1, p. 19-32, 2003.

CATHARINO, T. R. Matrimônio e maternidade. Considerações sobre as estratégias de patologização do corpo feminino. En: JACÓ VILELA, A.; CEREZO, A. C.; RODRIGUES, H. C. de B. (Org.). Clio-PsychéSubjetividae e História. Juiz de Fora: Clio Edições Eletrônicas, 2006, p. 188-1999.

ROSA RIVERO, A. El inútil combate entre la Psicología y su Historia. Revista de Historia de la Psicología, v. 29, n. 1, p. 31-66, 2008. ROSA, A; HUERTAS, J.; BLANCO, F. Haciendo historia para el futuro de la psicología. Anuario de psicología, v. 29, n. 1, p. 73-88, 1998. ROSE, N. A critical history of psychology. En: Inventing our Selves. Psychology, Power and Personhood. Cambridge: Cambridge University Press, 1996, p. 41-66

RUBIN, G. El tráfico de Mujeres: notas sobre la economía política del sexo. Revista Nueva Antropología, v. 8, p. 95-145, 1986.

SAAVEDRA, L.; NOGUEIRA, C. Memórias sobre o feminismo na psicologia: para a construção de memórias futuras. Memorandum, v. 11, 113-127, 2006.

SCOTT, J. El género: Una categoría útil para el análisis histórico. En: Cangiano, M.; Du Bois, L. (1993) (comp.). De mujer a género: teoría interpretación y practica feminista en las ciencias sociales. Buenos Aires: Centro Editor de América Latina. 1993, p. 17-50.

SHIELDS, P.; STEPHANIE, A. Passionate men, emotional women: Psychology constructs gender difference in the late 19th century. History of Psychology, v. 10, n. 2, p. 92-110, 2007.

SPUNG, H.; SPRUNG, L. Women in psychological history-forms of integration in psychology and womens lecturing activities at german psychology congesses 1904-1978. Revista de Historia de la Psicología, v. 17, n. 1/2, p. 185-202, 1996.

STOLKE, V. La mujer es puro cuento: la cultura del género. Revista Estudos Femeninos, v. 12, n. 2, p. 77-105, 2004.

VERA FERNANDEZ, J. Puente de plata para un provechoso encuentro entre la nueva historia y la psicología contemporánea. Pretextos para un diálogo necesario. Revista de Historia de la Psicología, v. 29, n. 1, p. 7-29, 2008.

VEZZETTI, H. Historias de la psicología: problemas, funciones y objetivos. Revista de Historia de la Psicología, v. 28, n. 1, p.147165, 2007.

VILANOVA, A. Enseñanza de la psicología: historia y problemas fundamentales. Cuadernos Argentinos de Historia de la psicología, v. 2, n. 1-2, p. 199-210, 1996.

VILANOVA, A. La Historia de la Psicología y su sentido curricular. Clepios, v. 3, n. 2, p. 18-23, 1997.

WATSON, R. The History of Psychology: A neglected area. American 
Psychologist, v. 15, p. 251-255, 1960.

WENTWORTH, P. The moral of her story: Exploring the philosophical and religious commitments in Mary Whiton Calkins' self-psychology. History of Psychology, v. 2, n. 2, p. 119-131, 1999.

WINKLER, M.; MAGAÑA, I.; WOOLF, X. Mujeres en la Historia de la Psicología: autorías y paradojas. Revista Latinoamericana de Psicología v. 33, n. 1, p. 23-37, 2001.

WINKLER, M.; MAGAÑA, I.; WOLF, X. Otra Historia De La Psicologia: Una Trilogía Femenina. Revista I nteramericana de Psicologia, v. 38, n. 1, p. 5-14, 2004.

WINKLER, M. Pioneras sin monumentos. Mujeres em Psicologia. Santiago de Chile: LOM, 2007.

\section{Endereço para correspondência}

Ana Elisa Ostrovsky

XX de septiembre, 1894, piso 3, dpto. "A", Código Postal 7.600, Mar del Plata, Argentina.

Endereço eletrônico: anaelios@hotmail.com

Recebido em: 31/03/2010

Aceito para publicação em: 03/08/2010

Acompanhamento do processo editorial: Ana Maria Jacó Vilela

\section{Notas}

* Licenciada en Psicología. Realiza sus estudios de doctorado en la Universidad Nacional de San Luis con beca CONICET.

${ }^{1}$ Nos referiremos a la reflexividad como la capacidad que tienen los seres humanos de tomarse a sí mismos como objeto de estudio. Capacidad de metaanálisis que establece una relación dialéctica e interdependiente entre los dos polos de conocimiento: el sujeto y el objeto, subrayando su historicidad y por lo tanto su mutabilidad. Dicho posicionamiento conlleva inevitablemente un cambio radical en la aproximación epistemológica naturalista tradicional. Véase: GERGEN, K. The social constructionist movement in modern psychology. American Psychologist, v. 40, p. 266-275, 1985. IBAÑEZ, T. La psicología social como dispositivo deconstruccionista. En: IBAÑEZ, T, El conocimiento de la realidad social. Barcelona: Sendai, 1989, p. 109-133.

${ }^{2}$ Hugo Klappenbach en el citado trabajo utiliza el concepto destino tomando como referencia la noción de destination empleada por George Ganguilhem con relación a la finalidad, al lugar de impacto del conocimiento en historia de la ciencia. Véase: CANGUILHEM, G. L'objet de I'histoire des sciences. En: CANGUILHEM, G. Etudes d'histoire et de philosophie des sciences, Paris: J. Vri, 1968, p.9-23

${ }^{3}$ Nos referimos a la escuela denominada así por el impacto de la revista Annales d'histoire économique et sociale fundada en 1929 por los historiadores Marc Bloch y Lucien Febvre. A lo largo de su publicación trascurrieron distintas generaciones que tuvieron como común denominador la incorporación a la historia de otras disciplinas como la geografía, la economía, la psicología social, la sociología y la antropología entre otras.

${ }^{4}$ Señalado en comunicación personal en el marco del Seminario "La historia social en debate (1950-2008)" Universidad de Buenos Aires- Centro Franco- Argentino de Altos Estudios (CFA) en el año 2008. 
${ }^{5}$ Nos referimos a espacios coyunturales en el marco de congresos y encuentros, pero con gran convocatoria y posibilidad de intercambio. Ejemplo de ello fue el Simposio: Género e Historia de la Psicología. En las XV Jornadas de Investigación y Tercer Encuentro de Investigadores en Psicología del Mercosur "Problemáticas actuales. Aportes desde la Investigación en psicología" llevado a cabo en Facultad de Psicología de la Universidad de Buenos Aires en agosto de 2008. Allí disertaron Lucía Rossi, Rosa Falcone y Ana María Jacó entre otras. Otro espacio que ejemplifica la tendencia fue la Mesa Redonda: Género e Historia Psi. En el marco del XIX Encuentro de Historia de la Psiquiatría, la Psicología y el Psicoanálisis realizado en la Facultad de Medicina de la Universidad de Buenos Aires en octubre del mismo año. 